\title{
SELETIVIDADE DO HERBICIDA TRIFLURALIN SOBRE O CRESCIMENTO INICIAL DA CANA-DE-AÇÚCAR
}

\author{
Fernando Tadeu de Carvalho ${ }^{1}$, Guilherme Manfrinatti Onohara ${ }^{2}$
}

\footnotetext{
${ }^{1}$ Prof. Dr. da UNESP Campus de Ilha Solteira (SP).

${ }^{2}$ Graduando em Agronomia na UNESP Campus de Ilha Solteira (SP).
}

RESUMO: O herbicida trifluralin aplicado em cana-de-açúcar pode atingir as gemas da cultura, dependendo da textura do solo e da quantidade de chuvas e, neste caso, não há informações sobre o nível de prejuízo que pode ocorrer. $\mathrm{O}$ objetivo do trabalho foi verificar a seletividade do trifluralin aplicado sobre as gemas nos toletes da cultura. $\mathrm{O}$ trabalho foi desenvolvido no município de Ilha Solteira, SP e o delineamento foi o de blocos inteiramente casualizados com 7 tratamentos e 4 repetições. As unidades experimentais foram constituídas de vasos plásticos de 50 litros com terra de campo. A variedade de canade-açúcar utilizada foi a RB 86-5453 e os tratamentos constituíram-se das concentrações de trifluralin aplicadas nas gemas das mudas: $100 \% ; 50 \% ; 10 \% ; 1 \% ; 0,1 \% ; 0,01 \%$ e uma testemunha sem aplicação. As avaliações de seletividade dos tratamentos foram realizadas através de testes de biometria. Observou-se que a seletividade do herbicida trifluralin à cana-de-açúcar é toponômica e que, ao entrar em contato com as gemas, nas concentrações testadas acima de $0,01 \%$, o herbicida causa reduções no crescimento aéreo e radicular das plantas. Concentrações inferiores a 1,0\% do herbicida permitem a germinação das gemas, porém retardam os seus crescimentos.

Palavras-chave: Controle químico. Saccharum spp. Fitotoxicidade. Biometria.

\section{SELECTIVITY OF THE TRIFLURALIN HERBICIDE ON INITIAL GROWTH OF SUGAR CANE}

\begin{abstract}
The trifluralin herbicide applied in sugar cane can reach the gems of culture, depending on the soil texture and the amount of rainfall and, in this case, there is no information on the level of injury that may occur. The objective of the work was to verify the selectivity of trifluralin applied on the buds in cuttings of the culture. The study was conducted in the municipality of Ilha Solteira, SP, Brazil, and the design was a completely randomized with 7 treatments and 4 replications. The experimental units consisted of plastic vessels of 50 liter with field soil. The variety of sugar cane used was RB 86-5453 and the treatments were the concentrations of trifluralin applied in the gems of seedlings: 100\%; $50 \% ; 10 \% ; 1 \% ; 0.1 \% ; 0.01 \%$ and an untreated control. The evaluations of treatment selectivity were made using biometric tests. It was observed that the selectivity of the trifluralin herbicide to sugar cane is by position and that, when in contact with the gems, in concentrations above $0.01 \%$, the herbicide causes reductions in shoot and root growth of the plants. Concentrations less than $1.0 \%$ of the herbicide allow the germination of the gems, but retard your growth.
\end{abstract}

Cultura Agronômica, Ilha Solteira, v.24, n.4, p.293-300, 2015 
Key words: Chemical control. Saccharum spp. Phytotoxicity. Biometrics.

\section{INTRODUÇÃO}

A seletividade dos herbicidas é analisada através dos índices de fitotoxicidade demonstrado pela cultura de tal forma que, quanto menor o índice mais seletivo é o herbicida. A análise da fitotoxicidade é baseada em redução da biomassa e/ou em alterações na coloração das plantas da cultura comparadas com as plantas da testemunha. Entende-se por seletividade a resposta diferencial de diversas espécies de plantas a um determinado herbicida. Via de regra, quanto maior a tolerância aos herbicidas da cultura em relação às plantas daninhas, maior é a segurança da aplicação (OLIVEIRA JÚNIOR.; CONSTANTIN, 2001).

A pontuação que é dada na avaliação da fitotoxicidade geralmente baseia-se em uma escala numérica dependendo do modelo proposto no trabalho. A escala de Frans et al. (1986), nos EUA, sugere notas de 0 a 100 e cita como base as seguintes caracterizações de fitotoxicidade: $0=$ nenhuma; $10=$ leve descoloração; $20=$ descoloração; $30=$ descoloração pronunciada com rápida recuperação; $40=$ descoloração pronunciada com recuperação; $50=$ descoloração pronunciada com recuperação lenta; $60=$ não recuperável; $70=$ grandes perdas na densidade; $80=$ plantas quase destruídas; $90=$ sobrevivem poucas plantas e $100=$ morte total da cultura.

Na Europa, a escala proposta por EWRC (1964) sugere notas de 1 a 9 e caracteriza os valores da seguinte forma: $1=$ nula; $2=$ muito leve; $3=$ leve; $4=$ fraca; $5=$ média; $6=$ quase forte; $7=$ forte; $8=$ muito forte e $9=$ destruição total. No Brasil tem sido muito comum o uso da escala de fitotoxicidade baseada em porcentagem onde $0 \%=$ nenhuma fitotoxicidade e $100 \%=$ morte total das plantas (SBCPD, 1995).

Os trabalhos de pesquisa que analisam os índices de seletividade e fitotoxicidade dos herbicidas são importantes, pois auxiliam na caracterização do potencial de uso dos produtos. $\mathrm{O}$ herbicida trifluralin é bastante utilizado na agricultura, em razão do baixo custo e de sua eficácia no controle de gramíneas, pertence ao grupo químico das dinitroanilinas, nome químico $\alpha, \alpha, \alpha$-trifluro-2,6-dinitro-n-n-dipropil-p-toluidina, com solubilidade em água de 0,3 ppm e possui translocação insignificante no solo sendo fortemente adsorvidos pelos colóides da argila (RODRIGUES; ALMEIDA, 2011).

O mecanismo de ação do herbicida trifluralin nas plantas é a inibição da divisão celular nos tecidos meristemáticos, inibindo a germinação das sementes e a formação de novas células na radícula e caulículo. Na cultura da cana-de-açúcar o herbicida é registrado para a aplicação em pré-plantio-incorporado com a ressalva de se evitar o contato do produto com os toletes (RODRIGUES; ALMEIDA, 2011), o que sugere que a seletividade seja toponômica.

Trabalhos de pesquisas têm demonstrado a redução do sistema radicular de cana-deaçúcar tratada com trifluralin. O herbicida é inclusive utilizado para inibir o crescimento de radicelas de cana-de-açúcar para dentro de sistemas de irrigação do tipo gotejadores 
enterrados (ORON et al., 1991; PIZARRO, 1996; DALRI et al., 2002).

A aplicação de herbicidas em cana-de-açúcar ocorre praticamente o ano inteiro, inclusive na época das chuvas (AZANIA et al., 2005) quando, dependendo da textura do solo e da quantidade precipitada, existe a possibilidade do trifluralin aplicado ser carreado até a região das gemas dos toletes e, neste caso, não há informações sobre o nível de prejuízo para a cultura.

O Brasil é o maior produtor de cana-de-açúcar do mundo com uma área de cultivo de 9,13 milhões de hectares na safra 2013/14, sendo a maior concentração no Estado de São Paulo com 4,7 milhões de hectares, segundo CONAB (2014). Considerando a importância da cultura para o Brasil e a possibilidade de prejuízos causados pelo herbicida, o presente trabalho foi desenvolvido, a fim de verificar, no crescimento inicial da cultura, a seletividade do herbicida trifluralin aplicado sobre as gemas nos toletes de cana-de-açúcar.

\section{MATERIAL E MÉTODOS}

O trabalho foi desenvolvido no Campus II da UNESP de Ilha Solteira, SP em ambiente aberto. $\mathrm{O}$ delineamento utilizado foi o de blocos inteiramente casualizados com sete tratamentos e quatro repetições. As unidades experimentais foram constituídas de vasos plásticos de 50 litros com terra de campo, textura argilosa com $45 \%$ de argila, $8 \%$ de silte e 47 de areia. A variedade de cana-de-açúcar utilizada foi a RB 86-5453. O plantio das mudas (Figura 1), tratadas e não tratadas, foi realizado em 17/06/2014 e os vasos foram irrigados diariamente e mantidos ao sol.
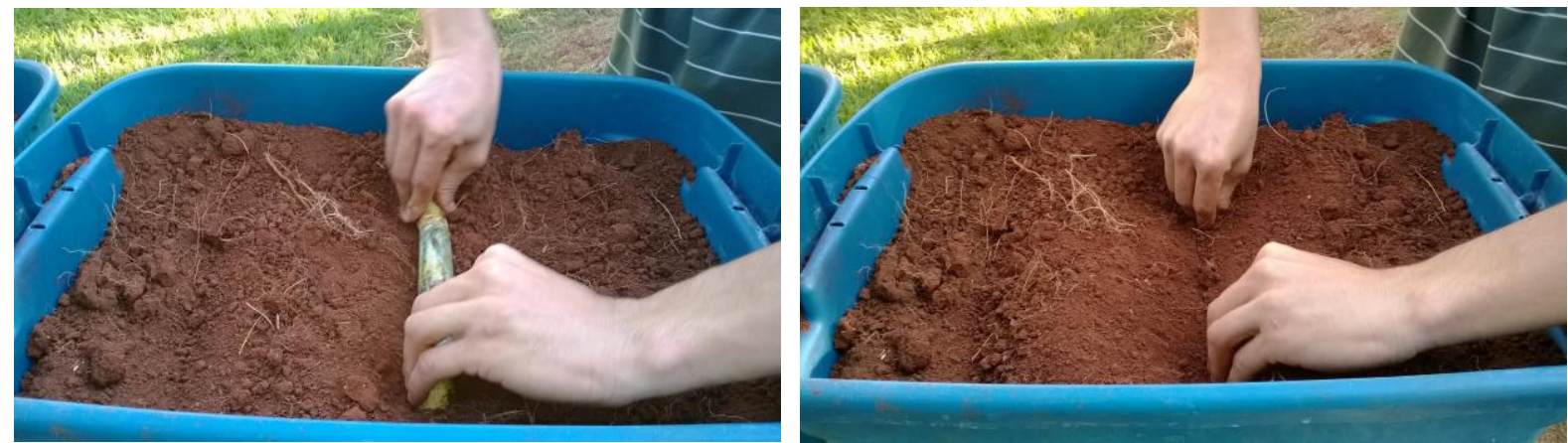

Figura 1. Plantio das mudas em vasos de 50 litros. Ilha Solteira, SP (2014).

Fonte: Dados do próprio autor.

Nas mudas que constituíram os tratamentos aplicados, o herbicida trifluralin foi aplicado sobre as gemas utilizando-se de um conta-gotas (Figura 2) nas concentrações de $100 \%$ (tratamento 2); $50 \%$ (tratamento 3); 10\% (tratamento 4); 1\% (tratamento 5); 0,1\% (tratamento 6); 0,01\% (tratamento 7) e testemunha sem aplicação (tratamento 1).

Cultura Agronômica, Ilha Solteira, v.24, n.4, p.293-300, 2015 


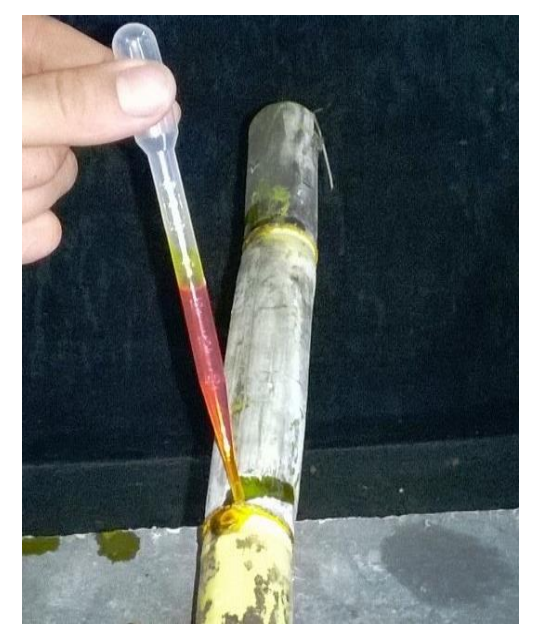

Concentração $100 \%$

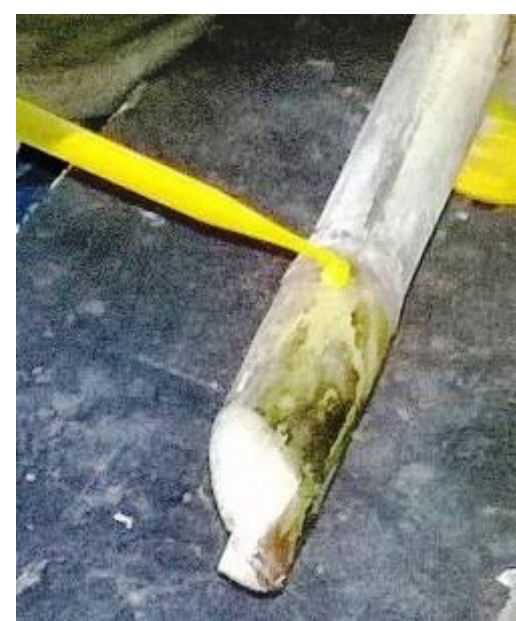

Concentração $10 \%$

Figura 2. Aplicação dos tratamentos nas gemas das mudas. Ilha Solteira, SP (2014). Fonte: Dados do próprio autor.

O herbicida estudado possui as seguintes características: ingrediente ativo: trifluralin, grupo químico: dinitroanilinas, concentração de ingrediente ativo: 450 g.L ${ }^{-1}$, formulação: concentrado emulsionável.

As avaliações de seletividade dos tratamentos foram realizadas aos 60 e 90 dias após a aplicação (DAA) dos tratamentos através de testes de biometria. Avaliou-se a altura e diâmetro das plantas aos 60 DAA e a altura, diâmetro e peso de raízes e da parte aérea das plantas aos 90 DAA. Os dados obtidos nas diferentes avaliações foram submetidos à análise de variância pelo teste $\mathrm{F}$, e, para comparação das médias utilizou-se o teste de Tukey a 5\% de probabilidade.

\section{RESULTADOS E DISCUSSÃO}

Os dados de crescimento das plantas aos 60 e 90 DAA estão apresentados na Tabela 1. Observa-se pelos dados de biometria que as plantas do tratamento Testemunha sem herbicida foram as que mais cresceram em todos os parâmetros e datas avaliados. Apesar do herbicida trifluralin ser fortemente adsorvidos pelos colóides da argila (RODRIGUES; ALMEIDA, 2011), deve-se considerar que, no presente trabalho, não houve interferência do solo já que o herbicida foi aplicado diretamente nas gemas antes do plantio das mudas.

A concentração que pode ser considerada como correspondente ou a que mais se aproxima da dose de campo é a de $1 \%$, se considerar-se a dose média de registro recomendada de 2,0 L.ha-1 (LORENZI et al., 2006) para um solo com 45\% de argila, aplicada em volume de calda de 200 L.ha $^{-1}$. Nesta concentração, de 1\%, observa-se que houve uma redução altamente significativa no crescimento das plantas, com relação à altura (9,0 e 75,0 cm, aos 60 e 90 DAA) o que correspondeu a uma redução de $91,1 \%$ aos 60 DAA e de 42,3\% aos 90 DAA e, com relação ao diâmetro (3,0 e 6,0 mm, aos 60 e 90 DAA) o que correspondeu a uma redução de $75,0 \%$ aos 60 DAA e de 53,8\% aos 90 DAA, em relação ao tratamento Testemunha sem herbicida. Este resultado de menor crescimento inicial das plantas de cana-de-açúcar é bastante prejudicial no sentido de tornar a cultura menos 
competitiva contra as plantas daninhas e menos tolerante às pragas e doenças.

$\mathrm{Na}$ concentração de $0,1 \%$ (dose aproximada de campo dividida por 10) observa-se também que houve redução significativa no crescimento das plantas, com relação à altura (41,0 e 100,0 cm, aos 60 e 90 DAA) o que correspondeu a uma redução de $59,5 \%$ aos 60 DAA e de 23,1\% aos 90 DAA e, com relação ao diâmetro $(6,0$ e 10,0 mm, aos 60 e 90 DAA) o que correspondeu a uma redução de 50,0\% aos 60 DAA e de $23,1 \%$ aos 90 DAA, em relação ao tratamento Testemunha sem herbicida.

Na concentração de $0,01 \%$ (dose aproximada de campo dividida por 100) observa-se também que houve reduções no crescimento das plantas, com relação à altura $(72,5$ e 125 $\mathrm{cm}$, aos 60 e 90 DAA) o que correspondeu a uma redução de $28,4 \%$ aos 60 DAA e de $3,8 \%$ aos 90 DAA (não significativa) e, com relação ao diâmetro $(9,0$ e 11,0 mm, aos 60 e 90 DAA) o que correspondeu a uma redução de $25,0 \%$ aos 60 DAA e de $15,4 \%$ aos 90 DAA, em relação ao tratamento Testemunha sem herbicida.

Nas concentrações de $10 \%$, 50\% e 100\% não houve germinação de gemas da cana-deaçúcar o que comprova que a seletividade é toponômica (por posição) e que deve ser evitado o contato do produto com os toletes, conforme recomenda Rodrigues e Almeida (2011).

Com relação ao peso de raízes e de parte aérea observa-se que o tratamento testemunha apresentou-se sempre superior aos tratamentos com o herbicida. No que diz respeito ao crescimento de raízes observou-se reduções, em relação à testemunha, de $87,7 \%$ no tratamento com $1,0 \% ; 40,6 \%$ no tratamento com $0,1 \%$ e $20,3 \%$ no tratamento com 0,01\% do herbicida. Tais resultados corroboram com Oron et al. (1991), Pizarro (1996) e Dalri et al. (2002) que utilizaram o herbicida trifluralin como inibidor do crescimento de raízes de plantas de cana-de-açúcar.

Tabela 1. Dados de biometria das plantas aos 60 e 90 DAA e peso de colmos e raízes aos 90 DAA. Ilha Solteira, UNESP (2014).

\begin{tabular}{|c|c|c|c|c|c|c|}
\hline \multirow[t]{2}{*}{ TRATAMENTOS } & \multicolumn{2}{|c|}{$\begin{array}{l}\text { Altura de Plantas } \\
\text { (cm) }\end{array}$} & \multicolumn{2}{|c|}{$\begin{array}{c}\text { Diâmetro de } \\
\text { Colmos (mm) }\end{array}$} & \multirow{2}{*}{$\begin{array}{c}\text { Peso de } \\
\text { Raízes } \\
(\mathrm{g})\end{array}$} & \multirow{2}{*}{$\begin{array}{c}\begin{array}{c}\text { Peso de } \\
\text { Colmos } \\
(\mathrm{g})\end{array} \\
90 \text { DAA }\end{array}$} \\
\hline & $60 \mathrm{DAA}$ & 90 DAA & 90 DAA & 60 DAA & & \\
\hline 1- Testemunha sem Herbicida & $101,3 \mathrm{a}$ & $130,0 \mathrm{a}$ & $69,0 \mathrm{a}$ & $12,0 \mathrm{a}$ & $13,0 \mathrm{a}$ & $89,5 \mathrm{a}$ \\
\hline 2- Trifluralin $(0,01 \%)$ & $72,5 \mathrm{~b}$ & $125,0 \mathrm{a}$ & $55,0 \mathrm{~b}$ & $9,0 \mathrm{~b}$ & $11,0 \mathrm{~b}$ & $66,5 \mathrm{ab}$ \\
\hline 3- Trifluralin $(0,1 \%)$ & $41,0 \mathrm{c}$ & $100,0 \mathrm{~b}$ & $41,0 \mathrm{c}$ & $6,0 \mathrm{c}$ & $10,0 \mathrm{~b}$ & $60,0 \mathrm{~b}$ \\
\hline 4- Trifluralin $(1,0 \%)$ & $9,0 \mathrm{~d}$ & $75,0 \mathrm{c}$ & $8,5 \mathrm{~d}$ & $3,0 \mathrm{~d}$ & $6,0 \mathrm{c}$ & $18,5 \mathrm{c}$ \\
\hline 5- Trifluralin (10\%) & $0,0 \mathrm{~d}$ & $0,0 \mathrm{~d}$ & $0,0 \mathrm{~d}$ & $0,0 \mathrm{e}$ & $0,0 \mathrm{~d}$ & $0,0 \mathrm{c}$ \\
\hline 6- Trifluralin (50\%) & $0,0 \mathrm{~d}$ & $0,0 \mathrm{~d}$ & $0,0 \mathrm{~d}$ & $0,0 \mathrm{e}$ & $0,0 \mathrm{~d}$ & $0,0 \mathrm{c}$ \\
\hline 7- Trifluralin (100\%) & $0,0 \mathrm{~d}$ & $0,0 \mathrm{~d}$ & $0,0 \mathrm{~d}$ & $0,0 \mathrm{e}$ & $0,0 \mathrm{~d}$ & $0,0 \mathrm{c}$ \\
\hline Média Geral & 31,96 & 61,43 & 24,79 & 4,29 & 5,71 & 33,50 \\
\hline Teste F (tratamentos) & $79,5 * *$ & $676,2 * *$ & $252,3 * *$ & $82,5 * *$ & $197,4 * *$ & $51,1 * *$ \\
\hline Coeficiente de Variação & $28,8 \%$ & $7,5 \%$ & $15,0 \%$ & $24,9 \%$ & $14,3 \%$ & $31,5 \%$ \\
\hline DMS (5\%) & 21,51 & 10,81 & 8,69 & 2,50 & 1,91 & 24,61 \\
\hline
\end{tabular}

Médias seguidas de letras iguais nas colunas, não diferem entre si ao nível de significância de 5\%, pelo teste de Tukey. DAA = Dias Após a Aplicação do herbicida.

No que diz respeito ao crescimento de colmos observou-se reduções, em relação à testemunha, de $79,3 \%$ no tratamento com $1,0 \% ; 33,0 \%$ no tratamento com $0,1 \%$ e $25,7 \%$ no 
tratamento com $0,01 \%$ do herbicida. Os resultados de redução de crescimento de colmos foram semelhantes aos de raízes.

\section{CONCLUSÃO}

Considerando as condições em que o trabalho foi desenvolvido, concluiu-se que a seletividade do herbicida trifluralin à cana-de-açúcar é toponômica e que, ao entrar em contato com as gemas, nas concentrações testadas acima de $0,01 \%$, este herbicida causa reduções no crescimento aéreo e radicular das plantas, permitindo a germinação das gemas apenas nas concentrações inferiores a 1,0\% do herbicida, mas com prejuízos significativos nos seus crescimentos.

\section{REFERÊNCIAS BIBLIOGRÁFICAS}

AZANIA, C. A. M.; ROLIM, J. C.; CASAGRANDE, A. A.; LAVORENTI, N. A.; AZANIA, A. A. P. M. Seletividade de herbicidas. III - aplicação de herbicidas em pósemergência inicial e tardia da cana-de-açúcar na época das chuvas. Planta daninha, Viçosa, v. 23, n. 4, p.489-495, 2005.

CONAB. Acompanhamento da Safra Brasileira - Cana-de-Açúcar - Safra 2014/15. $1^{\circ}$ Levantamento. Abril de 2014. Disponível em: http://www.Conab.gov.BR/OlalaCM S/uploads/arquivos/14_04_15_15_44_37_boletim_cana__ português_-1o_lev_-_14.pdf. Acesso em: 25 fev. 2015.

DALRI, A.; GARCIA, C.; ARAÚJO, M.; CRUZ, R.; DUENHAS, L. Uso da trifluralina para controle de intrusão de radicelas de cana-de-açúcar em gotejadores enterrados. In: CONGRESSO BRASILEIRO DE ENGENHARIA AGRÍCOLA, 31., 2002, Salvador. Resumos... Salvador: CONBEA, 2002. p. 1322-1325.

EWRC. Report of 3rd and 4rd meetings of EWRC. Comitee of methods in weed research. Weed Research, Oxford, England, v. 4, n. 1, p. 88. 1964.

FRANS, R.; TALBERT, R.; MARX, D. Experimental design and techniques for measuring and analyzing plant responses to weed control practices. In: CARPER, N. D. Research methods in weed science. 3.ed. Champaing: Southern Weed Science Society, 1986. p. 37.

LORENZI, H.; RODRIGUES, B. N.; FRANCO, D. A. S.; GAZZIERO, D. L. P.; COSTA, E. A. D.; ROMAN, E. S.; CARVALHO, F. T.; BLANCO, F. M. G.; PEREIRA, F. A. R.; CONSTANTIN, J.; ABUD, J. K.; NOLDIN, J. A.; DURIGAN, J. C.; VARGAS, L.; PENCKOWSKI, L. H.; NICOLAI, M.; MATALLO, M. B.; BIANCHI, M. A.; CORTEZ, M. G.; MOREIRA, M. S.; FLECK, N. G.; CHRISTOFFOLET, P. J.; VIDAL, R.; VICTÓRIA FILHO, R.; ARÉVALO, R. A. Manual de identificação e controle de plantas daninhas: plantio direto e convencional. 6. ed. Nova Odessa: Plantarum, 2006. 339 p.

Cultura Agronômica, Ilha Solteira, v.24, n.4, p.293-300, 2015 
OLIVEIRA JÚNIOR., R. S.; CONSTANTIN, J. Plantas daninhas e seu manejo. Guaíba: Agropecuária, 2001. 362 p.

ORON, G.; DEMALACH, J.; HOFFMAN, Z.; CIBOTARU, R. Subsurface microirrigation with effluent. Irrigation And Drainage Engineering, Reston, v. 117, n. 1, p.25-37, 1991.

PIZARRO, C. F. Riegos Localizados de Alta Frecuencia (RLAF). 3. ed. Bilbao: MundiPrensa, 1996. 513 p.

RODRIGUES, B. N.; ALMEIDA, F. S. Guia de herbicidas. 6. ed. Londrina: Edição dos autores, 2011. 697 p.

SOCIEDADE BRASILEIRA DA CIÊNCIA DAS PLANTAS DANINHAS (SBCPD). Procedimentos para instalação, avaliação e análise de experimentos com herbicidas. Londrina: SBCPD, 1995. 42 p. 
